\title{
Technical Aspects of Digitization of Cadastral Maps
}

\author{
Ram Kumar Sapkota \\ Survey Officer \\ Survey Department \\ ramksap@dos.gov.np \\ Ganesh Prasad Bhatta \\ Chief Survey Officer \\ Survey Department \\ ganesh.bhatta@dos.gov.np
}

\begin{abstract}
Mankinds of the 21st century are seeking for qualitative matters. They can't be satisfied only with the normal service; they expect value added service i.e. qualitative service. To cope the customer's satisfaction, almost all sectors are enhancing their performance and service delivery system using the information technology. Digitization of cadastral map is very essential for providing effective, reliable and qualitative cadastral services. Having the digitized cadastral maps and GIS database we can have a lot of advantages related to storage, management, retrieval, analysis, dissemination, update as well as IT enabled effective and reliable cadastral service delivery. In the present context of being scarcity of conventional instruments, ammonia paper and other accessories, it is crucial to enroll into this process and modernize land information in order to run with time, technology and international community.
\end{abstract}

\section{Keywords}

Digitization, Scanning, Geo-referancing, GIS, Seamless Database, Edge M atching, Computer based Cadastral Service Delivery

\section{Background}

Mankinds of the 21st century are seeking for qualitative matters. They can't be satisfied only with the normal service; they expect value added service i.e. qualitative service. To cope the customer's satisfaction, almost all sectors are enhancing their performance and service delivery system using the information technology. For this, service providers are adopting modern computer technology. B efore adopting the computer technology the most important step is to convert the analogue format data to the digital format in order to provide prompt, effective and quality service delivery. The digital conversion is possible basically by two methods. The first method is data entry of the analogue information in the designed and customized computer application and the next method is scanning of the analogue documents and further processing. In dictionary we can see, Digitizing is central to making a digital representation of geographic features, using raster or vector images, in a geographic information system i.e. the creation of electronic maps, either from various geographic and satellite images or by digitizing traditional paper maps. Digitization is also used to describe the process of populating databases with files or data. Therefore in our context, Digitization of cadastral map is the process of conversion of analogue(Paper/Cloth) maps to the digital form. More specifically it is the process of conversion of analogue map to vector map with its associate database.

\section{Introduction}

Cadastral map is a fundamental data that prescribes parcel numbers, land boundaries, classification of land and ownership of land parcels. It aims at defining and guaranteeing legal property boundaries and determining the area and perimeter to give information on the size and nature of land use. It is a very serious matter regarding to the property right of every citizen therefore providing computer based efficient and effective cadastral service is highly essential. Obviously, there could be two possible methods for computerizing cadastral services. The first method could be possible with the primary data acquisition i.e. survey and mapping with computer technology which definitely enhances quality of data 
(if perfectly done). But it may be a very long process that takes more time, money and efforts. The next method could be the digitization of analogue maps and preparation of geodatabase. This method could be effective, less time consuming and economic as well. Special care and precaution should be taken while doing digitization. A ctually, it is a secondary data acquisition of cadastral data through which each and every information of analogue maps are converted into digital form.

\section{Steps of Digitization}

Digitization of cadastral maps is the process of acquiring vector map from the existing analogue maps. It means source of vector data is the existing analogue maps. Therefore source must be clean, clear, unfold, non scratched and non shrinkaged. Practically it may not be possible to get such ideal cadastral maps in survey offices due to overuse of maps in day to day activities. Hence before scanning, the primary step should be tracing of analogue map.

\section{Tracing of analogue maps}

Definitely, quality of the product depends upon the source adopted. In order to achieve clean, clear, scanned image; scanning source must be fine. Therefore it should be taken as a necessary step prior

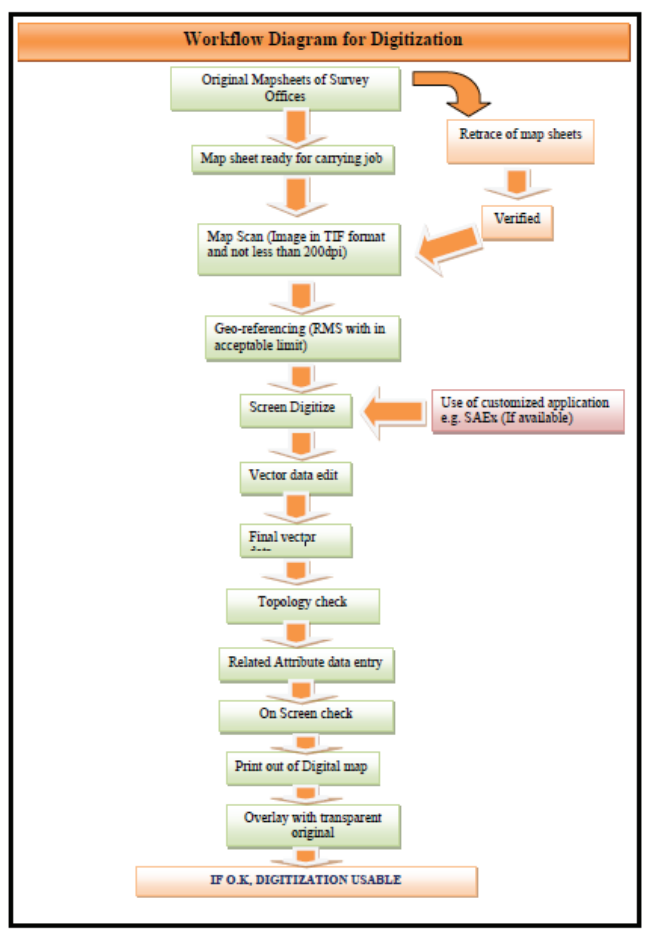

to scanning of cadastral map. Tracing should be done from the original cadastral sheet. If the original sheet itself is al so in poor condition, it is better to obtain the certified mapsfrom the general public or financial institution for tracing purpose. Tracing should be done using standard materials and then verified \& approved by the authorized personnel. In case the cadastral map on the clothing paper, even being fine, it should be strictly retraced on permatrace as such clothing material is not appropriate to fed into the scanner.

\section{Scanning}

Scanning is the next important step. A ctually it is the process of conversion of analogue maps to raster i $\mathrm{m}$ ages.

Scanning should be done with a roller scanner with at least 200 dpi (dots per inch) resolution in grey scale.

Scanning on 200 dpi resolution is equivalent to ground pixel size of $0.3175 \mathrm{~m}$ for 1:2500 map which is sufficient. Precaution should be taken to feed the traced cadastral map such that it will be completely vertically aligned and no warning will occur. The scanned cadastral maps will be better to store in "TIFF" format without compression. It is important to check the image with maximum zoom and diagonal check in order to ensure that there will be no distortion in the scanned image. $M$ aps should be properly strengthen and should be made dust $\&$ dirt free. Also, calibration of scanner should be properly done for better scanning results.

\section{Geo-referencing}

\subsection{G eo-referencing of G rid Sheets}

As, there are known co-ordinates of the four corners of grid in Grid Sheets. By the help of the known co-ordinates we can geo-reference the Grid Sheets. While inputting the co-ordinate of the corners of grid, the corner tics should be sharply identified. Using the four corner tics and $1^{\text {st }}$ degree polynomial transformation produced images sometimes has slight 
mismatch and gaps at the edge with other adjoining sheets. This may due to the wrong or displaced corner tics and warped sheet boundaries. This can be resolved by feeding the co-ordinate of the control points within the grid sheet and using $3^{\text {rd }}$ degree polynomial transformation to rectify the image. A fter geo-referencing the rectified images should have the R M S error less than the specified tolerance level.

\subsection{G eo-referencing of $F$ ree Sheets}

Geo-referencing of free sheet is a very difficult and challenging task. As there are no more known points in the sheets we can't feed the coordinates directly. To geo-reference the free sheet we have to identify the certain scattered points or details, than we have to go to field survey for finding out the coordinates of the identified points. Practically, it is very difficult and time consuming task. Instead of geo-referencing of free sheet from the real ground co-ordinate system, we can geo-reference with an arbitrary coordinate system. There could be two possible methods for this. One could be drawing a grid with fixed dimension on a free sheet; we can give arbitrary coordinate and geo-reference arbitrarily. This method may be quite complicated as there need to draw perfect grid manually on original map. A nother alternative method of geo referencing the free sheets is georeference with the help of "TIFF world file" (.tfw) file. Actually, it is a geo-referencing of scanned map pixels to an arbitrary coordinate system. Each scanned free sheet image in TIFF format are scaled to ground coordinate scale with a reference .tfw file. This is a text file containing the pixel reference and associated ground coordinate or arbitrary ground coordinate. The .tfw file format is readable for all GIS and CAD software. This method may be quite easy and convenient for geo referencing of cadastral maps with arbitrary coordinates.

\section{Digitization}

The scanned images after geo-referencing either ground coordinate system or arbitrary coordinate will be ready for digitization. A ctually digitization is the most important step through which vector data is generated from the raster data or scanned image. Digitization can be done automatically, semi-automatically and manual techniques. But cadastral map is the sensitive data related to property right and a single millimeter variation may cause a serious problem; manual or on screen digitization

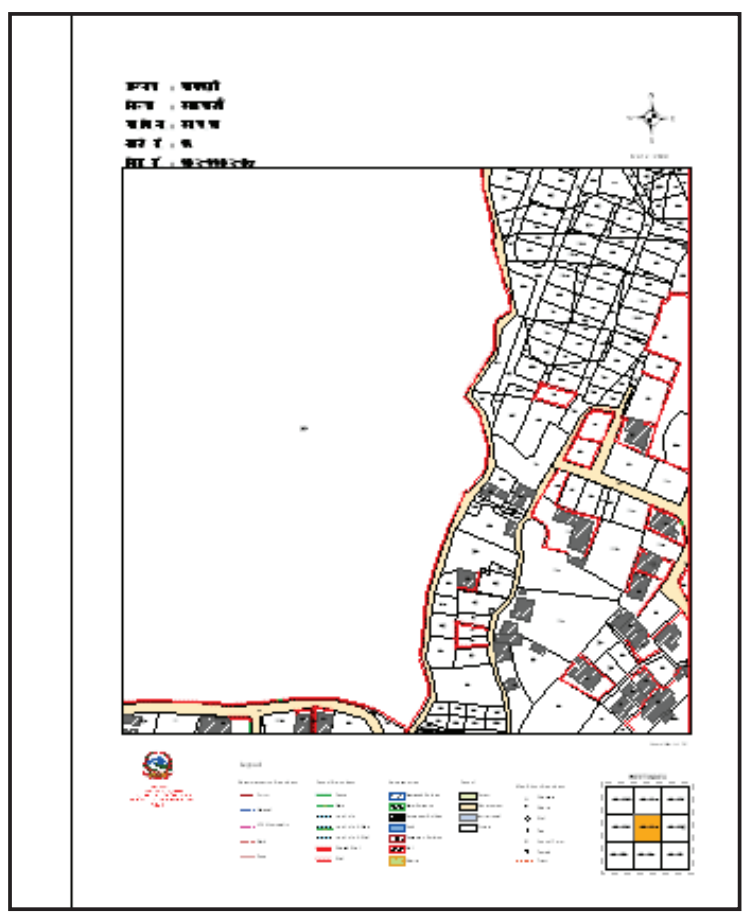

technique is the most appropriate technique as it is very accurate and reliable technique of digitization. Digitization can be done using various GIS and CAD softwares. It is more appropriate to use customized application based on A rcGIS to bring uniformity and consistency in digitization process. e.g. In our context SAEx (Spatial Application Extension), a customized application of ArcGIS software can be used as it contains certain feature class with definite fields with required characters. It is better to digitize after mosaicing the adjacent images so that edge matching problem can be minimized to some extent. Digitization should be seriously done by the experienced digitizers more preferably, the surveyors with GIS knowledge. While digitizing the image should be zoomed at least six times of the original and the digitizing lines should pass through the center width of the zoomed image. Special care should be taken for snapping within the tolerance on the node, vertex and edge in order to reduce topological errors. The digitized data should be topologically error free. The topology should be checked using the topology tools in GIS software. Digitization can also be understood as the digital tracing of the cadastral maps with mouse. While digitization each and every parcel should be seriously digitized in clockwise direction with minimum possible points. 


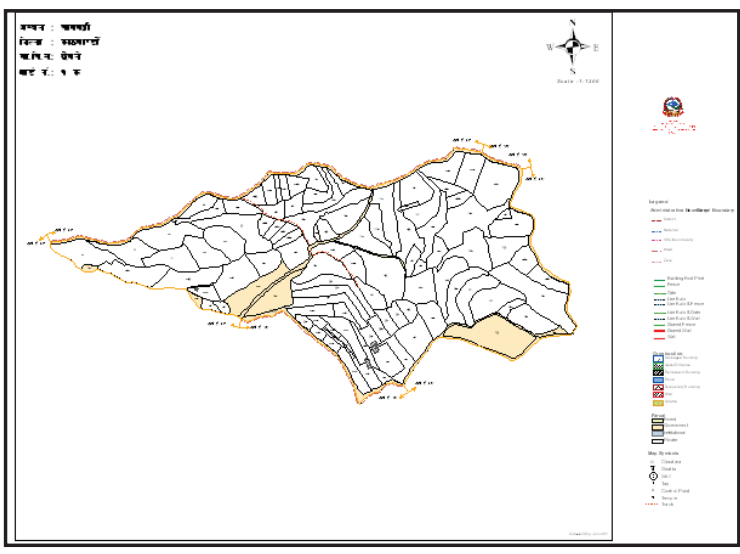

\section{Attribute Data E ntry}

A fter digitization and topological error check, the next step is the attribute data entry. In this step attribute data associated to individual parcel is entered in the attribute table. E.g.. parcel number, land use, land classification, VDC name/code, district name/code, ward no., Grid sheet number/ free sheet number, building type, boundary type etc. A rea and perimeter of each parcel with the feature class/shape file will be automatically calculated and other information related to parcel. A nd other information can be given manually. Feature class/ shape file can be created as per requirement with required fields and characters. If we are working under customized application e.g. SAEX we can give the attribute information as per the field in the feature class. Giving the attribute information related to the feature class, we can have the whole information in a single click. A ttribute should be used as per the standardized code (if available) to bring uniformity in data so that linking and merging of data will be possible in future.

\section{Digitized data check}

The digitized data should be thoroughly checked on computer screen to ensure the each and every parcel and other information of the cadastral image is correctly digitized. For this the vector data is overlaid over a scanned image. This can be taken as a step of Geometric check of the vector data generated. A fter that, each and every parcel numbers and their respective attribute information should be checked in a detailed way so that the parcel number and other attribute data are completely corrected. This can be taken as attribute check. Both checks should be done on computer screen as "H eads-up Checking" of digitized data.

\section{Print and Overlay Check}

A fter on screen data checking, the vector map should be printed out with a appropriate plotter on the original scale and sizei.e. A 0/A 1 of the source map so that each and every parcel and details can be checked one by one to ensure that the digitized data product and the original anal ogue maps coincides exactly and there will be no error in digitized data and could be used for computerized service delivery to the general public and other stake holders. Sometimes, the printed maps coincide only with the overlaid parcels and adjoining parcels but mismatch gradually with the far parcels. Therefore before printing, plotter should be well calibrated and adjusted. The final overlay check or final verification should be done by overlaying the printed maps over anal ogue maps using glass table to check overlay accurately and precisely.

\section{Digitization and G eodatabase U sable}

After all these abovementioned checks on the digitized data and geodatabase, the final product can be acceptable and usable for day to day cadastral activities in concerned offices. Special care and attention should be given for cent percent checking of the generated data although being complex, time consuming and tedious because small and minor mistakes in digitization may bring severe problems and complication during service delivery.

\section{Benefits:}

(1) Effective and Quality Service Delivery

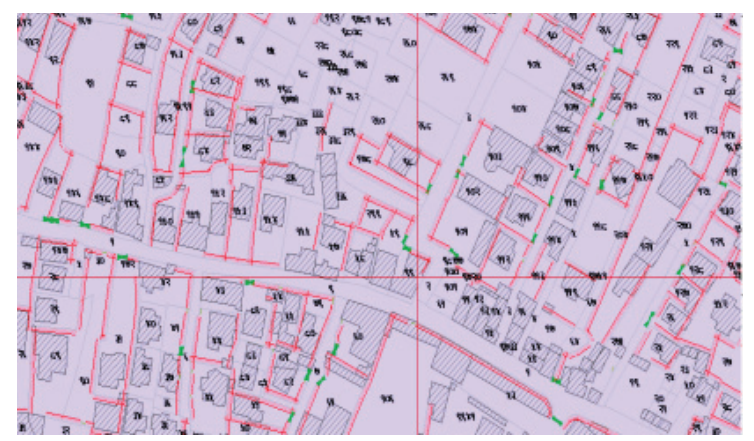

A fter digitization, effectiveness on service delivery will be increased and quality on service delivered will be enhanced. Service seekers will get prompt, transparent, reliable and consistent service delivery from survey offices. Parcel split, Parcel merge, $M$ ap Print can be done very accurately and precisely. The 




area of an individual parcel will always be same i.e. consistent results; which is almost impossible in traditional methods. Once area and dimension accepted it will be forever. There will never a

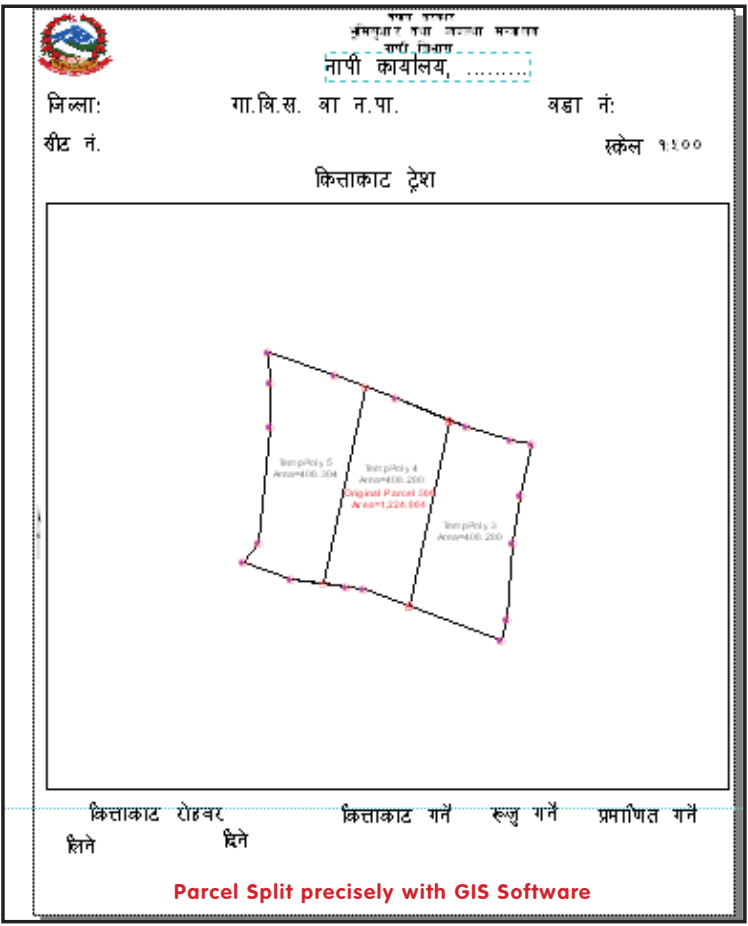

discrepancy in area and dimension even in a meter square and centimeter. A rea and other relevant information can be obtained from a single click. Public will able to get computer printed cadastral maps in portable size i.e.A4/A 3 size papers. A mmonia prints could be totally replaced and we can get rid from dependency on sunlight for map print. Parcel split trace (kittakat trace), Parcel trace (kitta trace), M ap print can be given in any scale and size.

\section{(2) Safe Storage and management of Cadastral Data}

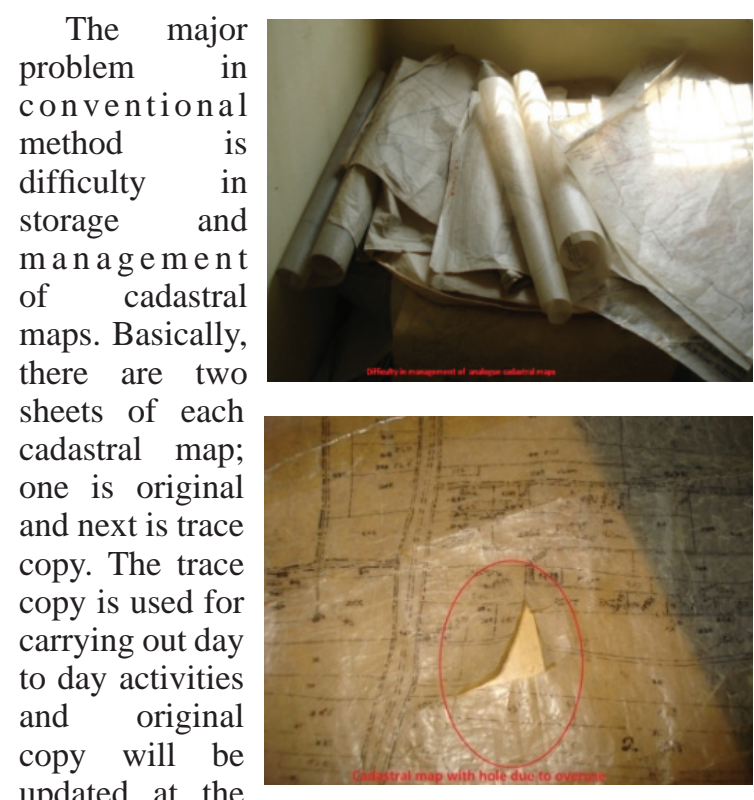
updated at the end of each day.

The maps will be wear and tear due to overuse and the maps will be faint, shrinkaged, stretched as well as mutilated too. There will be gradual deterioration of the cadastral maps. There is frequent need to retrace the cadastral maps. O riginal sheet, trace sheet and frequently traced trace sheets are difficult to store and manage even in a single or multiroom. This may al so create a confusion to identify the trace maps and update maps. A fter digitization all these issues can be easily addressed. A II the digitized data can be stored on a single hard disk or on a singl e computer. Backup in next computer or hard disk or DVD can be kept for safety of the data. Data will be retrieved from the computer and day to day activities will be done from a single chair and computer. There will be no need of tracing and retracing of cadastral maps.

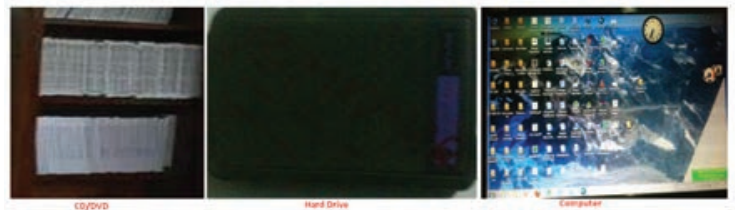




\section{(3) Working in G IS E nvironment}

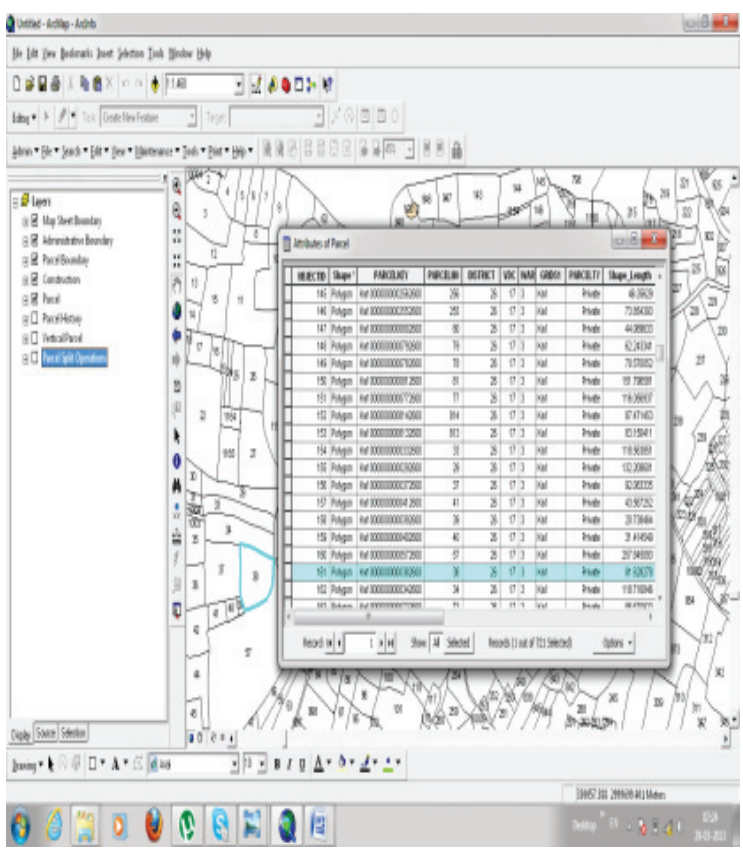

After digitization of cadastral maps, there will be a drastic change in the working environment i.e. from conventional environment to computerized GIS environment. Cadastral maps can be stored, managed, retrieved analyzed, disseminated and update within GIS environment. Using the GIS software and environment we can have various kinds of advantages. Various kinds of analysis can be easily done and can reduce the one day work in one minute. Various queries can be easily done needed for planning, policy making and other developmental activities. E. g.. how many parcels are less than $2 \mathrm{~A}$ nna 2 Paisa in certain specified area, how many parcels

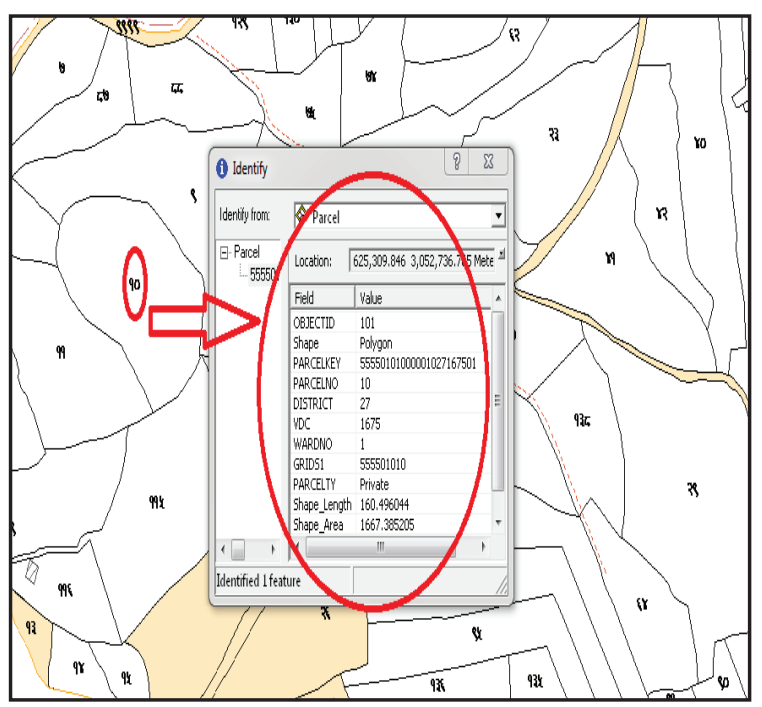

and how much area are covered by government or public in certain area, what is the buffer zone of road in the 15 meters expansion and how much area should be given compensation. A nalysis and visualization with various parameters can be easily done and useful information can be extracted.

\section{(4) Reduces $R$ epetition of work}

A fter having digitized data, there will no need of trace and retrace of cadastral maps time to time. There will no need of preparation of isolated file maps and parcel plans as the digitized data it can be zoomed in and out to any scale as per requirement. In traditional method, it is necessary to make file maps if we want to enlarge the parcel. If the parcel is small and difficult to split and mention parcel number, a file map is prepared in next separate permatrace which is difficult to store, manage and retrieve. In addition there will be more chances of introducing errors while making file maps. Digitized data will help to reduce such repetitive works.

\section{(5) R educes Duplication of work}

Cadastral data is the fundamental data and is highly essential in every development activities. E.g.. while construction or upgrading of road, irrigation, transmission line, railway, canal, drinking water, sewerage etc. it is very necessary to identify the parcel and area coverage of the alignment. And concerned organization will acquire and digitize the cadastral maps to have cadastral information. If we share or sell the prepared digitized data there will be no duplication of work and it will bring homogeneity on data and definitely reduces time, cost and effort.

\section{(6) Data L inkage}

With the digitized data we can link other relevant data and information. For example if we have scan fieldbooks images, plot register images, we can hyperlink the parcel with the respective fieldbook and plot register image so that we can also have field book and plot register information on a single click. It means all the associated documents can be linked and obtained from a single click. M oreover, we can have linkage with the other attribute data of $L$ and R evenue Office by LAN network generating common key on database. As a result, on a single click of parcel we can have information of area, perimeter, land use along with owner's name, owner's father's name and so on.

\section{(7) Information Sharing}

With the digitized data throughout the country helps 
to establish land Information System (LIS). The digitized data can be shared in uneditable or view only mode with various stakeholders' organizations, banks, financial institutions, and local bodies to assist in their respective activities. We can also generate good revenue from this information sharing. This can be done through local netw ork or web based network.

\section{Some Technical Issues}

Digitization of cadastral maps has many advantages. In the modern era of information communication technology, digitization of cadastral maps and providing computer based cadastral service is highly essential. However, there are some technical issues that should be addressed for computer cadastral services.

\section{(1) Area Difference between Legal Area and Digitized A rea}

A rea calculation from the analogue maps which we accept as a legal area are based on the traditional manual method whereas area calculation of the digitized cadastral data is based on modern computer methods. Naturally there will be discrepancies in area between digitized area and legal area. The legal area was calculated using computing scale and grid with approximation method and eye judgment techniques whereas area of digitized and cadastral map is calculated by the software itself based on digitized data. This is a common and worldwide issue after digitization.

\section{Possible solution}

The legal area and digitized area should be studied and analyzed. The first solution could be the digitized area will be compared to the legal area and a standard discrepancy could be made for acceptance. While calculating the legal area by the traditional method, two surveyors calculate the area of the same parcel, if the area difference calculated by two surveyors lie on the specified tolerance level, the first area is acceptable. Likewise we can accept the digitized area if it falls on the given specified tolerance level. M oreover, a study could be made between the area of digitized area and legal area and a new tolerance level could be defined. If the area between digitized and legal area falls on the defined tolerance, digitized area could be accepted and further activities could be done from digitized area. If the discrepancy doesn't fall in tolerance level, digitized data should be checked. If it is correct than the legal area from the analogue map should be checked. If necessary, field verification should also be done. The alternative solution could be the defining the tolerance level in percentage basis depending upon size of parcel. e.g. $0.5 \%$ for less than $500 \mathrm{~m}^{2}, 1 \%$ for more than $500 \mathrm{~m}^{2}$. M any countries adopt this technique to address this solution.

\section{(2) E dge M atching}

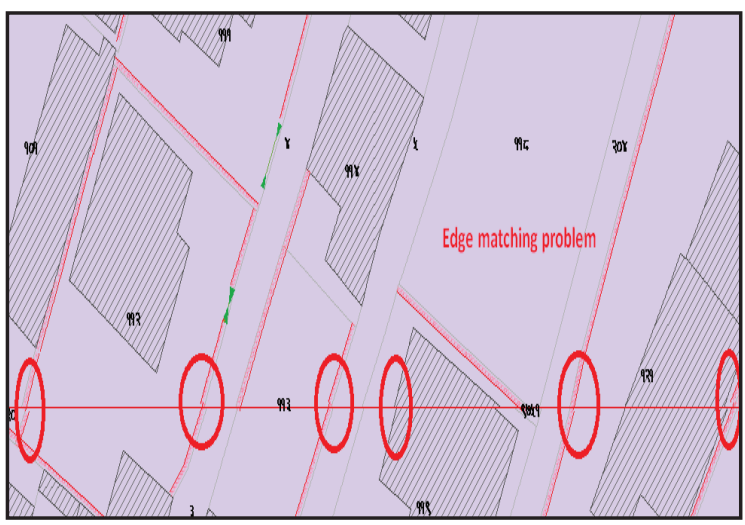

Itisanother serious issuefeltduring digitization of grid sheets and making seamless database. This problem arises when the same parcel lying in different sheets doesn't exactly coincide on the adjacent sheets. This problem may be due to shrinkage or stretched on the boundaries of original cadastral maps and improper geo-referencing of the sheets. This problem may be al so due to error in source or anal ogue maps. It means the parcel may not be edge matched while matching manually too i.e. due to problem in mapping or in re-tracing.

\section{Possible solution}

To minimizethis problem, a fresh traced copy (without that shrinkage and expand) analogue map should be used for scanning. While geo-referencing corner tics should be sharply identified and coordinated.

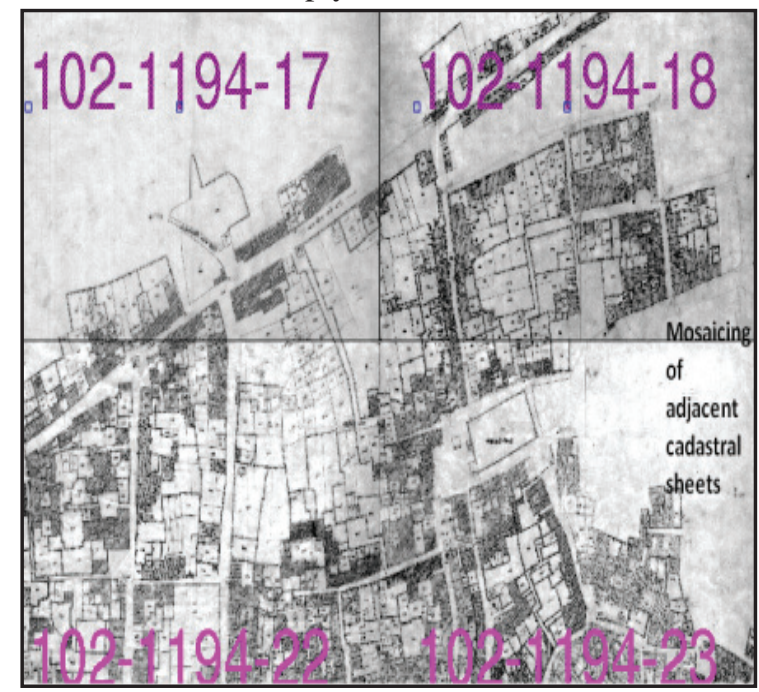


The next important step to reduce this problem is M osaicing the adjacent scanned image and clipping to meet parcel in the edge before digitization. Digitization should be done through the center of the edged parcel if the mismatch between them is less than the tolerance level. A Iternately, temporary solution could be tracing of that edged parcel from the analogue maps in a separate permatrace and then scan, geo-reference and digitize to carry out day to day activities.

\section{(3)A djustment of File M aps}

Due to constraint of zooming in or enlarge on the analogue maps due to its static nature, file maps are prepared when the parcel is small and difficult to split and write parcel number within it. They are prepared in large scale by enlarging proportionally. Theoretically, such file maps should have the same orientation, shape and area to the parent parcel. But practically, they may vary in shape and area in many cases due to various reasons. While digitization such file maps should also be adjusted to their respective parent parcel to make a complete integrate database but being variation in area and shape, it may difficult to do and may cause serious problem in many cases.

\section{Possible solution}

Rectifications of such file maps can be done according to legal document i.e. deed and then integrated to the parent database. Alternatively, as a temporary solution, a separate scanning of the filemap can be done and then referenced and digitized in a separate database. To manage and retrieve easily such separate database, hyperlink can be made in the database of parent parcel. Restriction for preparing file maps should be made after implementing digitized database.

\section{(4) M istakes in Parcel Split}

Sometimes, parcel split by a surveyor will be incorrect and inaccurate. Such mistake may be un intentional due to excessive work pressure, lack of sincerity or sometimes intentional due to wrong intention of concerned officials. Such parcels are clearly identified after digitization.

\section{Possible Solution}

Such parcel can be corrected according to the deed at the time of transaction of that parcel by splitting the parcel exactly as per mentioned in the deed.

\section{(5) Data Security}

Data security is another genuine issue after digitization. As mentioned earlier, all the digitized data can be stored and managed from a single computer or from a single hard drive. There is a maximum probability of transferring of data from Pen drive, DV D or other storage device so that such data could be misused and misinterpreted for various malfunctioning activities. Besides, data on computer or hard disk could be last or damaged due to virus or damage of hardware.

\section{Possible Solution}

For well management and security of the digitized data, data should be kept only on a single computer. i.e. on a server computer. Software should be run from client computer with LAN network. System Administrator should be allocated giving full responsibility of the data security and backup. Antivirus should be kept on server. Password of server should be given only to system administrator and office chief. USB and DVD-ROM of the server should be made disabled. In client computer, data should not be imported and every activity should be done directly using the database of server with the username and password provided by system administrator. Backup should be kept by system administrator in next storage device also. A third backup can be kept in respective department with the responsibility of authorized person. Server should be kept with special care in well air conditioning. Server with mirroring facilities should be used so that data can be recovered from next mirrored hard disk, in case, a running hard disk of server is damaged.

\section{Conclusion}

Finally, Digitization of cadastral map is very essential for providing effective, reliable and qualitative cadastral services. Digitization of cadastral map is very sensitive task. A mong the various types of maps, cadastral maps being related to the rights of property; its sensitivity should be seriously realized before digitization of cadastral maps. Skilled and well knowledged digitizers, preferably surveyors with cadastral knowledge, can only digitize cadastral maps in a qualititative manner. Having the digitized cadastral maps and GIS database we can have a lot of advantages related to storage, management, retrieval, analysis, dissemination, update and IT enabled effective and reliable cadastral service delivery. In the present context of being scarcity of conventional instruments, ammonia paper and other accessories, it is crucial to enroll into this process and modernize land information in order to run with time, technology and international community. 


\section{R eferences}

Byoungjun SEO, Jaejoon JEONG, J aebin LEE and Prof. Yongil KIM, Korea, Development of hybrid vectorizing software for Digitization of cadastral maps.

Channa, V.N. and Gupta Avneesh, Digitisation of cadastral maps: A case study for U ttar Pradesh.

Digitization of cadastral maps: As implemented in West B engal.

Singh, R. M., Bhopal, Computerized Cadastral Mapping: A Modern Technological Improvisation.

Tang Hong-Wai Conrad, Cheng Nga-Fong, Hong Kong Cadastral Survey System: A comparison and its implementation.
Technical Papers of D oLIA.

Thakur, Mr. Rakesh Kumar, GIS application in UP Bhumi Sudhar Nigam.

Upadhyaya, Alok, State-wide digital database and GIS of cadastral maps.

Wan Aziz., Majid. K, Sahrum, S. Skudai. Teng, C.B., Cadastral reform in M alaysia: A vision to the 2000 s.

Xiaoyong CHEN, Jie DU, Takashi DOIHARA, $G$ eneralization of cadastral map based on graphics matching J apan.

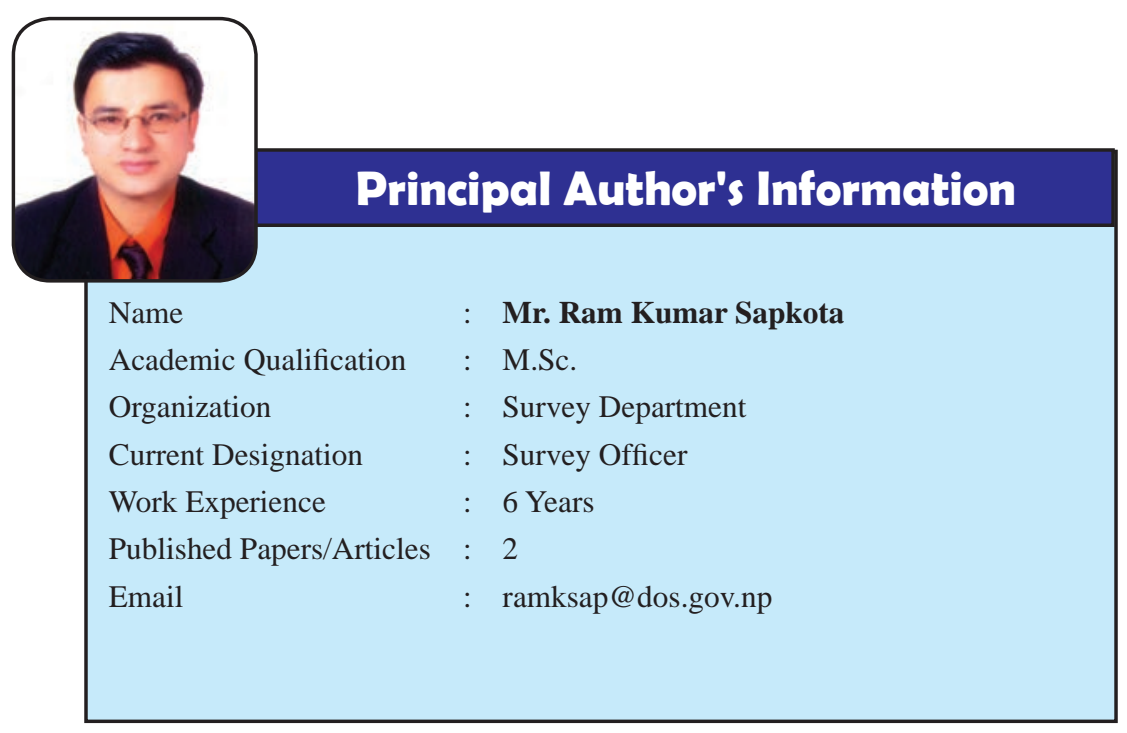

